\title{
Research on Dimensional Synthesis and Kinematics of Planar Mechanism
}

\author{
Bao-Lin YIN ${ }^{a}$, Bing-Bing YAN ${ }^{b}$, Chang-Chun $\mathrm{CHEN}^{\mathrm{c}}$, Chao-Qi $\mathrm{CHEN}^{\mathrm{d}}$, \\ Lin WANG ${ }^{\mathrm{e}}$
}

\author{
Department of Mechanical Engineering, Jiamusi University, Jiamusi, China \\ ayinblin@163.com, byanbingbing@126.com, Chencc515@163.com, d805793775@qq.com, \\ e1303403898@qq.com
}

Keywords: Planar Mechanism, Dimensional Synthesis, Nonlinear equation, Kinematics.

\begin{abstract}
The main idea in function generating synthesis of the planar mechanism is the design of a mechanism so that to provide a prescribed trajectory. In this paper a general method for dimensional synthesis is proposed. The synthesis equation is the nonlinear equation with three unknowns, and the equations are solved. Applications of these synthesized parameters, the kinematics of mechanism are established. And then, a numerical example is provided.
\end{abstract}

\section{Introduction}

Generally, to attempt to fulfill the specifications imposed in linkage design entails a long process. It is mainly due to the complexity of the mechanical systems and the difficulty of developing mathematical tools involving all the parameters taking part. Often, the analyst's experience and judgment predominates upon the information obtained from mathematical methodologies. In these cases, the analyst is obliged to repeat the process several times using his intuition until a suitable solution is obtained. Kinematic synthesis of planar linkages provides valuable tools to help the analyst to take decisions, playing an important role in the design of mechanisms and this importance increases as more performance requirements are demanded [1]. All synthesis problems generate drawbacks with similar characteristics involving a large number of nonlinear equations that are difficult to solve. When this occurs, the formulation may lead to a greater number of equations than the number of unknowns [2,3]. In the paper [4], the kinematic synthesis of Stephenson mechanisms for motion generation was carried out. And concerning the homotopy methods two approaches, means via the Bezout number and the BKK bound, had been compared. The paper [5] proposed a case-based approach, i.e. couples of trajectories and dimensions of a given structure mechanism, using a neural net.

Thus, the designer can carry out a large number of simulations efficiently, quickly and with low cost. However, due to the real world complexity, not all cases in mechanical design synthesis have been formulated and some of them remain unsolved.

In this paper a general method for dimensional synthesis of planar mechanism is proposed. Through analysis geometric relationship of the mechanism, synthesis equations are described. With three precision points, deriving the equations governing the problem leads to three couple equations. And the equations are solved. Applications of these synthesized parameters, the kinematics of mechanism are established. And then, a numerical example is provided.

\section{Description of the Planar Six-bar Mechanism}

The schematic of the planar six-bar mechanism is shown in Fig. 1. The mechanism are six-bar kinematic chains, connected by five revolute joints at point $O, A, B, C$ and $M$, and two prismatic joints at $D$. The revolute joint $A$ is actuated and the other joints are passive. A reference frame $O-x y z$ is attached to the base at point $O$. The geometric dimensions of the mechanism are defined as: $A B=L_{1}, B C=C M=$ $C O=L_{2}, A O=L_{3}, M P=L_{4}$. Special geometric relationship result to special trajectory of point $M$ and point $P$. 


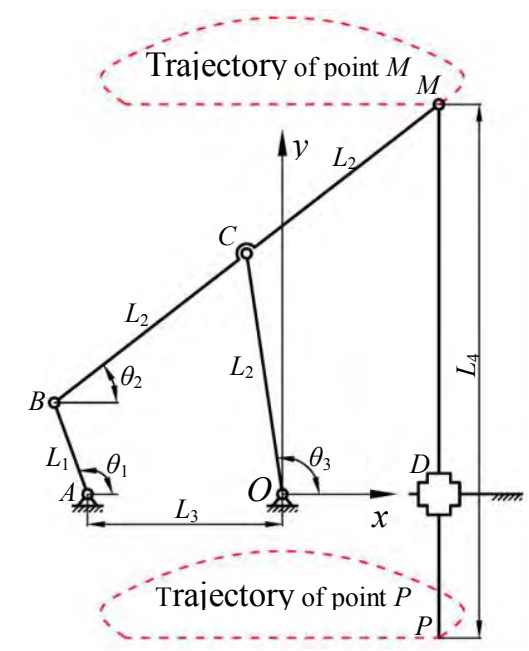

Fig. 1 Schematic of the Planar Six-bar Mechanism

\section{Synthesis Equations}

In Fig. 1, the position of $M$ in the $O-x y$ coordinate frame can be expressed as $x_{\mathrm{M}}$ and $y_{\mathrm{M} i}$

$$
\begin{aligned}
& x_{\mathrm{M}}=L_{1} \cos \theta_{1}+2 L_{2} \cos \theta_{2}-L_{3}, \\
& y_{\mathrm{M}}=L_{1} \sin \theta_{1}+2 L_{2} \sin \theta_{2} .
\end{aligned}
$$

Using triangular relations, Eq. 1 can be written as

$$
-L_{1}^{2}+4 L_{2}^{2}+L_{3}^{2}+x_{\mathrm{M}}^{2}+y_{\mathrm{M}}^{2}+2 L_{3} x_{\mathrm{M}}-4 L_{2}\left(L_{3}+x_{\mathrm{M}}\right) \cos \theta_{2}-4 L_{2} y_{\mathrm{M}} \sin \theta_{2}=0 .
$$

The position of $M$ in the $O-x y$ coordinate frame also can be expressed as

$$
\begin{aligned}
& x_{\mathrm{M}}=L_{2} \cos \theta_{3}+L_{2} \cos \theta_{2}, \\
& y_{\mathrm{M}}=L_{2} \sin \theta_{1}+L_{2} \sin \theta_{2} .
\end{aligned}
$$

Using triangular relations, Eq. 3 can be written as

$$
x_{\mathrm{M}}^{2}+y_{\mathrm{M}}^{2}-2 L_{2} x_{\mathrm{M}} \cos \theta_{2}-2 L_{2} y_{\mathrm{M}} \sin \theta_{2}=0 .
$$

From Eq. 2 and Eq. 4, the synthesis equation of the mechanism can be expressed as

$$
\begin{aligned}
F: & t_{1} L_{1}^{4}+t_{2} L_{2}^{4}+t_{3} L_{3}^{4}+t_{4} L_{1}^{2} L_{2}^{2}+t_{5} L_{1}^{2} L_{3}^{2}+t_{6} L_{2}^{2} L_{3}^{2}+t_{7} L_{1}^{2} L_{3}+t_{8} L_{2}^{2} L_{3}+t_{9} L_{3}^{3}+t_{10} L_{1}^{2} \\
& +t_{11} L_{2}^{2}+t_{12} L_{3}^{2}+t_{13} L_{3}+t_{14}=0 .
\end{aligned}
$$

where $t_{i}$ can be expressed as

$$
\begin{aligned}
& t_{1}=t_{3}=x_{\mathrm{M}}^{2}+y_{\mathrm{M}}^{2}, t_{2}=16\left(x_{\mathrm{M}}^{2}+y_{\mathrm{M}}^{2}\right), t_{4}=-8\left(x_{\mathrm{M}}^{2}+y_{\mathrm{M}}^{2}\right), \\
& t_{5}=-2\left(x_{\mathrm{M}}^{2}+y_{\mathrm{M}}^{2}\right), t_{6}=8\left(x_{\mathrm{M}}^{2}-y_{\mathrm{M}}^{2}\right), t_{7}=-t_{9}=-8\left(x_{\mathrm{M}}^{3}+x_{\mathrm{M}} y_{\mathrm{M}}^{2}\right), \\
& t_{8}=32\left(x_{\mathrm{M}}^{3}-x_{\mathrm{M}} y_{\mathrm{M}}^{2}\right), t_{10}=-6 x_{\mathrm{M}}^{4}-4 x_{\mathrm{M}}^{2} y_{\mathrm{M}}^{2}+2 y_{\mathrm{M}}^{4}, \\
& t_{11}=24 x_{\mathrm{M}}^{4}-48 x_{\mathrm{M}}^{2} y_{\mathrm{M}}^{2}-8 y_{\mathrm{M}}^{4}, t_{12}=22 x_{\mathrm{M}}^{4}+24 x_{\mathrm{M}}^{2} y_{\mathrm{M}}^{2}+2 y_{\mathrm{M}}^{4} \\
& t_{13}=24 x_{\mathrm{M}}^{5}+32 x_{\mathrm{M}}^{3} y_{\mathrm{M}}^{2}++8 x_{\mathrm{M}} y_{\mathrm{M}}^{4}, t_{14}=9 x_{\mathrm{M}}^{6}-19 x_{\mathrm{M}}^{4} y_{\mathrm{M}}^{2}+11 x_{\mathrm{M}}^{2} y_{\mathrm{M}}^{4}+y_{\mathrm{M}}^{6} .
\end{aligned}
$$

Eq. 5 is the nonlinear equation with three unknowns, $L_{1}, L_{2}$ and $L_{3}$. And each precision point corresponds to an equation. 


\section{Synthesis of Mechanism with Three Parameters}

The main idea in function generating synthesis of the planar six-bar mechanism is the design of a mechanism so that to provide a prescribed trajectory. As an example of the synthesis equation of the mechanism, three position of $M$ in the $O$-xy coordinate frame are given as: $M_{1}=(0,100), M_{2}=(0,82)$, and $M_{3}=(-40,82)$. The simultaneous equations in Eq. 5 are a set of three non-linear equations. The roots for $L_{i}$ are given in Table 1 . There are 24 solutions, and in which there are 6 real roots.

Tab.1 Solutions of $L_{i}$ for the Synthesis Equations $\left(\times 10^{2}\right)$

\begin{tabular}{|c|c|c|c|c|c|c|c|}
\hline No. & $L_{1}$ & $L_{2}$ & $L_{3}$ & No. & $L_{1}$ & $L_{2}$ & $L_{3}$ \\
\hline 1,2 & \pm 0.20151 & 0.51038 & 0.40642 & 13,14 & $\begin{array}{c} \pm(0.25243 \\
-0.10679 * \mathrm{I})\end{array}$ & $\begin{array}{c}-0.48752 \\
-0.00261 * \mathrm{I}\end{array}$ & $\begin{array}{c}0.27519 \\
+0.11642 * \mathrm{I}\end{array}$ \\
\hline 3,4 & \pm 0.20151 & -0.51038 & 0.40642 & 15,16 & $\begin{array}{c} \pm(0.25243 \\
-0.10679 * \mathrm{I})\end{array}$ & $\begin{array}{c}0.48752 \\
-0.00261 * \mathrm{I}\end{array}$ & $\begin{array}{c}0.27519 \\
-0.11642 * \mathrm{I}\end{array}$ \\
\hline 5,6 & \pm 0.52229 & 0.53234 & -0.15680 & 17,18 & $\begin{array}{c} \pm(0.01033 \\
-0.04482 * \mathrm{I})\end{array}$ & $\begin{array}{c}0.23213 \\
+0.7469 * \mathrm{I}\end{array}$ & $\begin{array}{c}0.39999 \\
+1.73506 * \mathrm{I}\end{array}$ \\
\hline 7,8 & \pm 0.52229 & -0.53234 & -0.15680 & 19,20 & $\begin{array}{c} \pm(0.01033 \\
-0.04482 * \mathrm{I})\end{array}$ & $\begin{array}{c}-0.23213 \\
+0.74694 * \mathrm{I}\end{array}$ & $\begin{array}{c}0.39999 \\
-1.73506 * \mathrm{I}\end{array}$ \\
\hline 9,10 & $\pm(0.25243$ & $\begin{array}{c}0.48752 \\
+0.00261 * \mathrm{I}\end{array}$ & $\begin{array}{c}0.27519 \\
+0.11642 * \mathrm{I}\end{array}$ & 21,22 & $\begin{array}{c} \pm(0.01033 \\
-0.04482 * \mathrm{I})\end{array}$ & $\begin{array}{c}-0.23213 \\
-0.74694 * \mathrm{I}\end{array}$ & $\begin{array}{c}0.39999 \\
+1.73506 * \mathrm{I}\end{array}$ \\
\hline 11,12 & $\begin{array}{c} \pm(0.25243 \\
-0.10679 * \mathrm{I})\end{array}$ & $\begin{array}{c}-0.48752 \\
+0.00261 * \mathrm{I}\end{array}$ & $\begin{array}{c}0.27519 \\
-0.11642 * \mathrm{I}\end{array}$ & 23,24 & $\begin{array}{c} \pm(0.01033 \\
-0.04482 * \mathrm{I})\end{array}$ & $\begin{array}{c}0.23213 \\
-0.74694 * \mathrm{I}\end{array}$ & $\begin{array}{c}0.39999 \\
-1.73506 * \mathrm{I}\end{array}$ \\
\hline
\end{tabular}

\section{Kinematics of the Planar Mechanism}

The position of $C$ in the $O-x y$ coordinate frame can be expressed as $x_{\mathrm{C}}$ and $y_{\mathrm{C}}$

$$
x_{\mathrm{C}}=-L_{3}+L_{1} \cos \theta_{1}+L_{2} \cos \theta_{2}=L_{2} \cos \theta_{3} \text {, }
$$$$
y_{\mathrm{C}}=L_{1} \sin \theta_{1}+L_{2} \sin \theta_{2}=L_{2} \sin \theta_{3} \text {. }
$$

Eliminating $\theta_{3}$, and Eq. 7 can be written as

$$
2 L_{2}\left(L_{1} \cos \theta_{1}-L_{3}\right) \cos \theta_{2}+2 L_{1} L_{2} \sin \theta_{1} \sin \theta_{2}+\left(L_{1}^{2}+L_{3}^{2}-2 L_{1} L_{3} \cos \theta_{1}\right)=0 .
$$

The half-angel tangent relationships can be expressed as

$$
\sin \theta_{2}=\frac{2 \tan \left(\theta_{2} / 2\right)}{1+\tan ^{2}\left(\theta_{2} / 2\right)}, \cos \theta_{2}=\frac{1-\tan ^{2}\left(\theta_{2} / 2\right)}{1+\tan ^{2}\left(\theta_{2} / 2\right)}
$$

The four-bar mechanism $A B C O$, is the crank-rocker mechanism, and $0<\theta_{2}<\pi$ 。 Substituting Eq. 9 into Eq. 8, yields

$$
\theta_{2}=2 \arctan \left(\frac{-u_{2}+\sqrt{u_{2}^{2}-4 u_{1} u_{3}}}{2 u_{1}}\right)
$$

where $u_{i}$ can be expressed as

$$
\begin{aligned}
& u_{1}=L_{1}^{2}+L_{3}^{2}-2 L_{1} L_{3} \cos \theta_{1}-2 L_{1} L_{2} \cos \theta_{1}+2 L_{2} L_{3}, \\
& u_{2}=2 L_{1} L_{2} \sin \theta_{1}, \\
& u_{3}=L_{1}^{2}+L_{3}^{2}-2 L_{1} L_{3} \cos \theta_{1}+2 L_{1} L_{2} \cos \theta_{1}-2 L_{2} L_{3} .
\end{aligned}
$$

Given the drive parameter $\theta_{1}$ and the dimensional parameters $L_{1}, L_{2}$ and $L_{3}$, the $\theta_{2}$ can be solved form Eq. 11. And then, Eq. 1 can be solved, and then the position of $M$ can be determined by back-substitution. 


\section{Numerical Example for Kinematics}

Applications of these synthesized parameters, $L_{1}=20 \mathrm{~mm}, L_{1}=50 \mathrm{~mm}, L_{3}=40 \mathrm{~mm}$. The trajectory of the point $M$ is depicted in Fig. 2. Therefore, the synthesis equations and the kinematics of the planar mechanism are minimal.

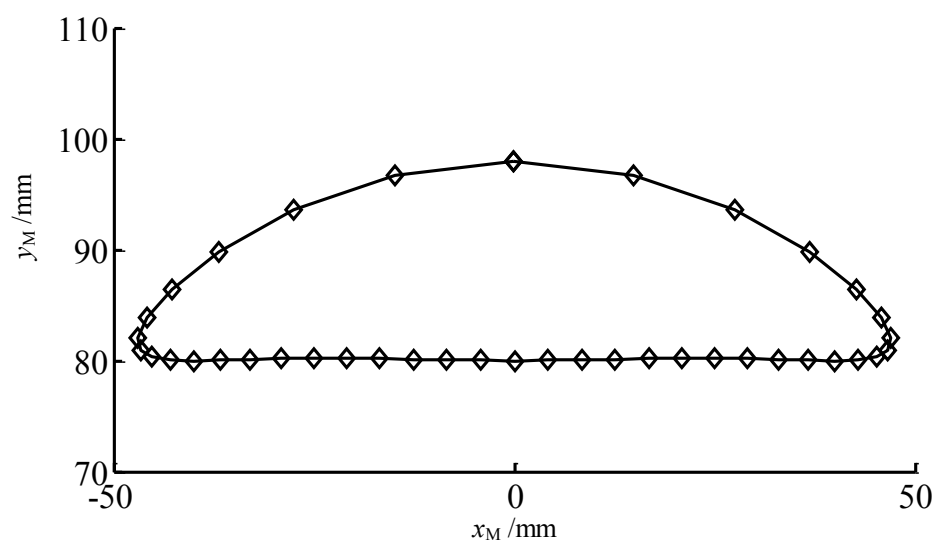

Fig. 2 Trajectory of the Point $M$

\section{Conclusion}

In this paper, the dimensional synthesis the planar mechanism has been successfully approached. Firstly, the position analysis was carried out, and synthesis equation of the mechanism was established. The synthesis is the nonlinear equation with three unknowns. The synthesis equation was solved with three positions, and 24 solutions for the dimensional parameters were achieved. And then, kinematics of the planar mechanism was established. Lastly, the numerical example for kinematics was carried out. Therefore, the synthesis equations and the kinematics of the planar mechanism are minimal.

\section{Acknowledgement}

This work was supported by science and technology research projects of Heilongjiang Province Office of Education (Item Number 12531671), and college students' innovation and entrepreneurship training projects of Heilongjiang Province (Item Number 201310222047).

\section{References}

[1]Y. M. Moon and S. Kota. Automated synthesis of mechanisms using dual-vector algebra, Mechanism and Machine Theory. 37 (2002) 143-166.

[2]R. Sancibrian, P. Garcia, F. Viadero and A. Fernandez. A general procedure based on exact gradient determination in dimensional synthesis of planar mechanisms, Mechanism and Machine Theory. 41 (2006) 219-229.

[3]R. Sancibrian, P. Garcia, F. Viadero and A. Fernandez. Gradient-based optimization of path synthesis problems in planar mechanisms, Mechanism and Machine Theory. 49 (2004) 839-856.

[4]H. Schreiber, K. Meer and B. J. Schmitt. Dimensional synthesis of planar Stephenson mechanisms for motion generation using circlepoint search and homotopy methods, Mechanism and Machine Theory. 37 (2) (2002) 717-737.

[5]A. Vasiliu and B. Yannou. Dimensional synthesis of planar mechanisms using neural networks: application to path generator linkages, Mechanism and Machine Theory. 36 (2001) 299-310. 